\title{
Anisotropic pseudo-potential for polarized dilute quantum gases
}

\author{
Andrei Derevianko \\ Department of Physics, University of Nevada, Reno, Nevada 89557
}

(Dated: October 27, 2018)

\begin{abstract}
Anisotropic pseudopotential relevant to collisions of two particles polarized by external field is rigorously derived and its properties are investigated. Such low-energy pseudopotential may be useful in describing collective properties of dilute quantum gases, such as molecules polarized by electric field or metastable ${ }^{3} P_{2}$ atoms polarized by magnetic field. The pseudopotential is expressed in terms of reactance $\left(\mathrm{K}^{-}\right)$matrix and derivatives of Dirac delta-function. In most applications it may be represented as a sum of traditional spherically-symmetric contact term and anisotropic part. The former contribution may be parameterized by a generalized scattering length. The anisotropic part of pseudopotential may be characterized by off-diagonal scattering length for dipolar interactions and off-diagonal scattering volume for quadrupolar interactions. Two-body matrix element of the pseudopotential in a basis of plane waves is also derived.
\end{abstract}

PACS numbers: 03.75.Fi, 34.20.Gj

\section{INTRODUCTION AND PROBLEM SETUP}

The concept of pseudo-potential, i.e. full interparticle interaction being replaced by some less complicated "effective" potential, plays an important role in many subfields of physics [1]. In particular, properties of traditional Bose-Einstein condensates (BEC) may be well understood just in terms of a delta-function potential, with its strength determined by a single parameter - scattering length [2, 3] which characterizes low-energy scattering between two particles. A rigorous derivation of pseudo-potential for spherically-symmetric interactions has been carried out by Huang and Yang [4]. Here I extend their derivation to anisotropic interactions. I also evaluate a matrix element of the derived anisotropic pseudo-potential in the basis of plane waves; this matrix element may be useful in studies of many-body properties of quantum degenerate gasses.

Unusual collective properties of bosons and fermions with anisotropic interactions have generated a considerable interest over the last few years, see e.g. Refs. 5, 6, 7, 8, 9, 10, 11, 12, 13, 14. Below I enumerate several systems where the results of my analysis may be applicable. First, Yi and You [5] considered an application of strong electric field to an atomic condensate. The electric field induces atomic electric dipoles and thus anisotropic dipole-dipole interactions between the atoms. Another novel systems where the anisotropic interactions dominate at large separations are heteronuclear molecules 87. Here an application of electric field is required to freeze the rotations of the molecules and to align the intrinsic molecular dipole moments with the field. Magnetic dipole-dipole interactions are present even for well-studied alkali-metal atoms. These interactions may be amplified for more complex atoms like europium and chromium [15, 16, 17] with larger mag-

*Electronic address: andrei@physics.unr.edu netic momenta of the ground atomic state. The influence of such magnetic dipolar interactions on the condensate properties was discussed in Ref. [9]. New systems where the anisotropy of interactions may be also of interest are metastable ${ }^{3} P_{2}$ alkaline-earth atoms placed in external magnetic field. Here the long-range forces are dominated by interactions of atomic quadrupoles [18]. It should be noted that the application of external magnetic or electric field is important in all these examples - the field fixes quantization axis and a condensate may be described in terms of a single order parameter.

In all the enumerated examples the collision process may be formalized using Fig. 11. Here we show a pair of identical particles interacting in the presence of external uniform field. The $z$-axis is chosen along the direction of the field and angle $\theta$ determines orientation of collision (interatomic) axis $\hat{\mathbf{r}}$ with respect to the field. At large separations $r$ the particles are polarized along the direction of the field. In the most general case, as a result of a collision, a change in polarization may occur (e.g. dipole moment of a molecule could end up pointing in the direction opposite to the field). We will disregard these non-adiabatic collisions. Then the interaction between the particles may be described by a unique potential $V(r, \theta)$. Without loss of generality, this axially-symmetric potential may be expanded into Legendre polynomials $P_{L}(\cos \theta)$

$$
\begin{aligned}
V(r, \theta) & =V_{\mathrm{sph}}(r)+V_{\text {anis }}(r, \theta), \\
V_{\text {anis }}(r, \theta) & =\sum_{L=2,4, . .} V_{L}(r) P_{L}(\cos \theta) .
\end{aligned}
$$

Here $V_{\mathrm{sph}}(r)$ and $V_{\text {anis }}(r, \theta)$ are spherically-symmetric $(L=0)$ and non-spherical contributions respectively. Although all even $L$ contribute, at sufficiently large $r$ the anisotropic contribution may be dominated by a single $L$ term. In particular, we will focus on two practically interesting cases - dipolar $(L=2)$

$$
V_{\text {anis }}(r, \theta) \rightarrow V_{\mathrm{DD}}=\frac{C_{3}}{r^{3}} P_{2}(\cos \theta), r \rightarrow \infty,
$$


and quadrupolar $(L=4)$

$$
V_{\text {anis }}(r, \theta) \rightarrow V_{\mathrm{QQ}}=\frac{C_{5}}{r^{5}} P_{4}(\cos \theta), r \rightarrow \infty
$$

interactions. In the above equations constants $C_{L+1}$ parameterize strengths of interactions and are proportional to the squares of respective multipole moments (e.g. molecular dipole moment).

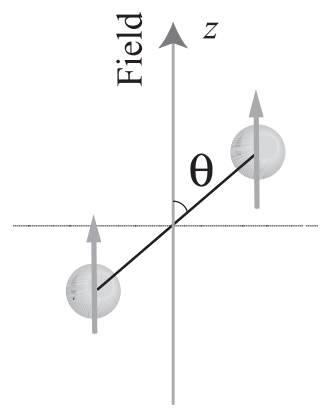

FIG. 1: Geometry of collision process. At large separations colliding particles are polarized along external field. During the collision the particles are assumed to follow a unique adiabatic potential.

Realistic interaction potentials $V(\mathbf{r})$ are singular at small interparticle separations and this singularity leads to well-known problems [19] in formulating perturbative expansion for many-body properties. In particular, matrix element of the interaction in basis of free-particle is divergent. To remedy this problem, the full interaction potential $V$ is usually replaced by a pseudopotential $\hat{\mathcal{V}}$. A rigorous derivation of pseudo-potential for sphericallysymmetric interactions has been carried out by Huang and Yang [4]. Here I extend their method to anisotropic interactions.

Previously, for anisotropic dipolar interactions, Yi and You [5] proposed the following pseudopotential

$$
\hat{\mathcal{V}}_{\mathrm{DD}}^{\mathrm{YY}}=g \delta(\mathbf{r})+\frac{C_{3}}{r^{3}} P_{2}(\cos \theta) .
$$

Here the first term is related to spherically-symmetric part of the full potential (2) and the second contribution is simply the long-range dipolar interaction (3). This pseudopotential has been employed in a large number of studies of properties of BECs with dipolar interactions, see, e.g. [5, 6, 7, 8, 9, 10, 11, 12]. Although straightforward to work with in applications, the pseudo-potential (5) has certain shortcomings. For example, it is not valid in the vicinity of resonances. Pseudo-potential derived here resolves these shortcomings.

The goal of this work is to consistently develop a pseudo-potential method for non-spherical interaction potentials. It will be required that two-body wavefunctions obtained with the pseudo-potential $\hat{\mathcal{V}}$ and full original potential $V$ to be equal at large interparticle separations. The derivation of this pseudo-potential is carried out in Section II. Certain properties of the derived pseudopotential are discussed in Section [II] and we specialize the discussion to dipolar and quadrupolar interactions in Section IV. Matrix element of the pseudo-potential in free-particle basis is evaluated in Section V. Finally, Appendix contains a derivation of certain low-energy limits of K-matrix in the Born approximation.

\section{ANISOTROPIC PSEUDO-POTENTIAL}

We consider a solution of the Schrödinger equation for a relative motion of two particles interacting through a potential $V(\mathbf{r})$

$$
\frac{\hbar^{2}}{2 \mu}\left(\nabla^{2}+k^{2}\right) \Phi(\mathbf{r})=V(\mathbf{r}) \Phi(\mathbf{r})
$$

where $\mu$ is reduced mass of the pair and $\mathbf{k}$ is the relative linear momentum. We assume that at sufficiently large separations $r>r_{c}, r^{2} V(\mathbf{r}) \rightarrow 0$ for any direction $\hat{r}$. We also assume that the particles are contained in some large volume with characteristic size much larger than the extent of the potential $r_{c}$. Some arbitrary boundary conditions may be imposed on the surface of the enclosing volume. At $r \gg r_{c}$ the wavefunction $\Phi(\mathbf{r})$ may be expanded in free-particle solutions

$$
\Phi_{\infty}(\mathbf{r})=\sum_{l m}\left(\alpha_{l m} j_{l}(k r)-\beta_{l m} n_{l}(k r)\right) Y_{l m}(\theta, \varphi)
$$

where $j_{l}(k r)$ and $n_{l}(k r)$ are spherical Bessel and Neumann functions respectively and $\alpha_{l m}$ and $\beta_{l m}$ are integration constants.

Following Ref. 四 the pseudo-potential is determined by acting with $\frac{\hbar^{2}}{2 \mu}\left(\nabla^{2}+k^{2}\right)$ on the asymptotic form (7)

$$
\begin{aligned}
& \hat{\mathcal{V}} \Phi_{\infty}(\mathbf{r})= \\
& \quad-\frac{\hbar^{2}}{2 \mu} \sum_{l m} \beta_{l m} Y_{l m}(\theta, \varphi) \frac{(2 l-1) ! !(l+1)}{k^{l+1}} \frac{\delta(r)}{r^{l+2}} .
\end{aligned}
$$

Thus the original potential $V(\mathbf{r})$ is replaced by a sum over "lumped" multipole sources placed at $\mathbf{r}=0$. To complete the construction of the pseudo-potential, we need to determine coefficients $\beta_{l m}$ in terms of $\Phi(\mathbf{r})$. First we relate the integration constants $\alpha_{l m}$ and $\beta_{l m}$ by requiring the complete solution $\Phi$ to be regular at $r=0$

$$
\beta_{l m}=\sum_{l^{\prime} m^{\prime}} \mathcal{K}_{l m}^{l^{\prime} m^{\prime}} \alpha_{l^{\prime} m^{\prime}}
$$

Here $\mathcal{K}_{l m}^{l^{\prime} m^{\prime}}$ are the elements of the reactance (or $\mathrm{K}^{-}$) matrix used to parameterize multi-channel scattering [20]. It is worth noting that the entire dependence of the pseudopotential on the original potential will be encapsulated in matrix elements of the K-matrix.

Let us review some properties of the K-matrix. First it is related to more familiar scattering matrix $S$ via $S=$ 
$(1+i \mathcal{K})(1-i \mathcal{K})^{-1}$ and further to transmission or $\mathrm{T}$ matrix through $S=1-i T$. For low-energy collisions $\mathcal{K} \approx-\frac{1}{2} T$. K-matrix is real and symmetric

$$
\mathcal{K}_{l m}^{l^{\prime} m^{\prime}}(k)=\mathcal{K}_{l^{\prime} m^{\prime}}^{l m}(k) .
$$

For identical bosons (fermions) only even (odd) partial waves need to be considered. The K-matrix is diagonal in $l$ and $m$ for spherically-symmetric potentials

$$
\left[\mathcal{K}_{\mathrm{sph}}\right]_{l m}^{l^{\prime} m^{\prime}}=\delta_{l l^{\prime}} \delta_{m m^{\prime}} \tan \delta_{l},
$$

where $\delta_{l}$ is the phase shift for partial wave $l$. Compared to spherically-symmetric case, anisotropic potentials additionally couple different partial waves. For example, dipolar interactions, Eq. (3), couple $s$ and $d$ waves so that $\left[\mathcal{K}_{\mathrm{DD}}\right]_{00}^{20} \neq 0$ and quadrupolar interactions (4) couple $s$ and $g$ waves. The scalar part $V^{\mathrm{sph}}(r)$ of the potential (2) assures non-vanishing $\mathcal{K}_{00}^{00}$. In practice, K-matrix for non-spherical interactions may be found from a solution of coupled radial equations [21]. In particular, it may be shown that for potentials parameterized by Eq. (2), $\mathcal{K}_{l m}^{l^{\prime} m^{\prime}} \propto \delta_{m m^{\prime}}$, i.e. the K-matrix is diagonal with respect to magnetic quantum numbers. In Appendix, I derive some elements of K-matrix in the Born approximation for dipolar and quadrupolar interaction.

At this point we related the integration constants $\alpha_{l m}$ and $\beta_{l m}$ via elements of K-matrix, Eq. (9). Further, in the low-energy limit $k r_{\mathrm{c}} \ll 1$, the integration constants $\alpha_{l m}$ may be expressed in terms of $\Phi_{\infty}(\mathbf{r})$ 化

$\alpha_{l m}=\frac{1}{2^{l} l !} \frac{1}{k^{l}}\left[\left(\frac{d}{d r}\right)^{2 l+1}\left(r^{l+1} \int Y_{l m}^{*}(\Omega) \Phi_{\infty}(\mathbf{r}) d \Omega\right)\right]_{r=0}$

Finally, combining equations (8)-(12) we arrive at a generalization of pseudo-potential $\hat{\mathcal{V}}$ for anisotropic interactions

$$
\hat{\mathcal{V}} \Phi(\mathbf{r})=-\frac{\hbar^{2}}{M} \sum_{l m l^{\prime} m^{\prime}} \xi_{l m}^{l^{\prime} m^{\prime}}(k) \quad\left(\hat{v}_{l m}^{l^{\prime} m^{\prime}} \Phi(\mathbf{r})\right),
$$

where

$$
\begin{aligned}
& \hat{v}_{l m}^{l^{\prime} m^{\prime}} \Phi(\mathbf{r})=\frac{(2 l) !(l+1)}{2^{l+l^{\prime}} l^{\prime} ! l !} Y_{l m}(\theta, \varphi) \frac{\delta(r)}{r^{l+2}} \times \\
& {\left[\left(\frac{d}{d r}\right)^{2 l^{\prime}+1}\left(r^{l^{\prime}+1} \int Y_{l^{\prime} m^{\prime}}^{*}(\Omega) \Phi(r, \Omega) d \Omega\right)\right]_{r} }
\end{aligned}
$$

pseudo-potential of Huang and Yang [4] is subsumed in this equation. Indeed, for spherically-symmetric interactions the K-matrix is diagonal in $l, l^{\prime}$ and $m, m^{\prime}$ and is expressed in terms of conventional phase shifts, Eq. (11). Upon substitution of Eq. (11) into pseudopotential (13) we recover as a limiting case the result of Ref. [4]. As the pseudo-potential of Huang and Yang [4] the anisotropic pseudopotential is non-Hermitian and velocity dependent.

The pseudo-potential (13) may be separated into spherically-symmetric $\hat{\mathcal{V}}_{\text {sph }}$ and anisotropic $\hat{\mathcal{V}}_{\text {anis }}$ parts

$$
\hat{\mathcal{V}}=\hat{\mathcal{V}}_{\text {sph }}+\hat{\mathcal{V}}_{\text {anis }},
$$

where

$$
\hat{\mathcal{V}}_{\mathrm{sph}}=-\frac{\hbar^{2}}{M} \sum_{l m} \xi_{l m}^{l m}(k) \hat{v}_{l m}^{l m}
$$

and

$$
\hat{\mathcal{V}}_{\text {anis }}=-\frac{\hbar^{2}}{M} \sum_{(l m)>\left(l^{\prime} m^{\prime}\right)} \xi_{l m}^{l^{\prime} m^{\prime}}(k)\left(\hat{v}_{l m}^{l^{\prime} m^{\prime}}+\hat{v}_{l^{\prime} m^{\prime}}^{l m}\right) .
$$

In simplifying the anisotropic part we used symmetry property (10) of K-matrix.

Let us focus first on the spherically-symmetric part of the pseudopotential and in the following section we will consider anisotropic part of the pseudopotential for dipolar and quadrupolar interactions. We expect that as in traditional BECs of dilute atomic gasses with sphericallysymmetric interactions, the effect of $\hat{\mathcal{V}}_{\text {sph }}$ on collective properties will be determined mainly by s-wave contri.bution, i.e. $l=0, m=0$ term in Eq. (17)

$$
\hat{\mathcal{V}}_{\mathrm{sph}} \approx-\frac{\hbar^{2}}{M} \frac{\mathcal{K}_{00}^{00}}{k} \hat{v}_{00}^{00} .
$$

It may be shown that for realistic potentials the following low-energy limit is finite

$$
a_{s s}=-\lim _{k \rightarrow 0} \frac{\mathcal{K}_{00}^{00}(k)}{k} ;
$$

this quantity is a generalized scattering length. With this definition, the truncated pseudopotential reduces to

$$
\hat{\mathcal{V}}_{\mathrm{sph}} \Phi(\mathbf{r}) \approx 4 \pi \frac{\hbar^{2}}{M} a_{s s} \delta(\mathbf{r}) \frac{\partial}{\partial r}(r \Phi(\mathbf{r})),
$$

$\xi_{l m}^{l^{\prime} m^{\prime}}(k)=\frac{\mathcal{K}_{l m}^{l^{\prime} m^{\prime}}}{k^{l+l^{\prime}+1}}$,

and $M=2 \mu$ is a mass of the collision partner.

\section{SOME PROPERTIES OF PSEUDOPOTENTIAL}

The derived anisotropic pseudopotential (13) is one of the main results of this work. The spherically-symmetric

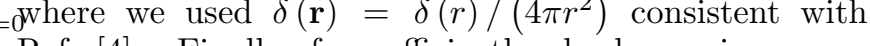

Ref. [⿰氵⿴囗十). Finally, for sufficiently slowly-varying wave(1f:olnction, $|d \log \Phi / d \log r| \ll 1$, we recover conventional contact pseudo-potential

$$
\hat{\mathcal{V}}_{\mathrm{sph}} \approx 4 \pi \frac{\hbar^{2}}{M} a_{s s} \delta(\mathbf{r}) .
$$

widely employed in studies of BECs.

Having discussed spherically-symmetric part of the pseudo-potential, in the following section we consider anisotropic part of pseudopotential (18) for dipolar and quadrupolar interactions. 


\section{DIPOLAR AND QUADRUPOLAR INTERACTIONS}

At this point we derived anisotropic pseudo-potential, Eq.(8). We separated the pseudopotential into spherically-symmetric and anisotropic contributions. We found that the spherically-symmetric contribution reduces to familiar contact term (20), widely employed in studies of Bose condensates; the only modification being an introduction of generalized scattering length (19). In this section we focus on the anisotropic contribution and illustrate some of its properties for dipolar and quadrupolar interactions of identical bosons. These interactions were defined in the introductory Section as potentials that at large separations $r$ are dominated by

$$
V_{\text {anis }}^{\mathrm{DD}}(r, \theta) \rightarrow \frac{C_{3}}{r^{3}} P_{2}(\cos \theta)
$$

for dipolar interactions and

$$
V_{\text {anis }}^{\mathrm{QQ}}(r, \theta) \rightarrow \frac{C_{5}}{r^{5}} P_{4}(\cos \theta)
$$

for quadrupolar interactions.

Anisotropic interactions mix different partial waves $(l m)$ and $\left(l^{\prime} m^{\prime}\right)$ via off-diagonal elements of K-matrix. From examining Eq. A2 in Appendix, one may determine that angular selection rules lead to coupling of $s$ and $d$ waves for dipolar interactions and $s$ and $g$ waves for quadrupolar interactions. In the following we assume that the dominant anisotropic effect on condensate properties arises due to these particular couplings. Therefore,

$$
\begin{aligned}
& \hat{\mathcal{V}}_{\text {anis }}^{\mathrm{DD}} \approx-\frac{\hbar^{2}}{M} \frac{\mathcal{K}_{00}^{20}}{k^{3}}\left(\hat{v}_{00}^{20}+\hat{v}_{20}^{00}\right) \\
& \hat{\mathcal{V}}_{\text {anis }}^{\mathrm{QQ}} \approx-\frac{\hbar^{2}}{M} \frac{\mathcal{K}_{00}^{40}}{k^{5}}\left(\hat{v}_{00}^{40}+\hat{v}_{40}^{00}\right) .
\end{aligned}
$$

For dipolar interactions it may be shown (Refs. [22, 23] and Appendix of this paper) that the following lowenergy limit is finite

$$
a_{s d}^{\mathrm{DD}}=-\lim _{k \rightarrow 0} \frac{\mathcal{K}_{00}^{20}(k)}{k} .
$$

We will call this quantity off-diagonal scattering length. Similarly, for quadrupolar interactions we may introduce off-diagonal scattering volume

$$
a_{s g}^{\mathrm{QQ}}=-\lim _{k \rightarrow 0} \frac{\mathcal{K}_{00}^{40}(k)}{k^{3}} .
$$

In the Appendix we employ Born approximation and find

$$
\begin{aligned}
& a_{s d}^{\mathrm{DD}} \approx \frac{1}{12 \sqrt{5}} \frac{M}{\hbar^{2}} C_{3}, \\
& a_{s g}^{\mathrm{QQ}} \approx \frac{2}{7 !} \frac{M}{\hbar^{2}} C_{5} .
\end{aligned}
$$

It is worth noting that the above results are valid only away from resonances. In a general case one has to find off-diagonal scattering length or volume by solving corresponding scattering problem.

Finally the total truncated pseudopotential is given by

$$
\hat{\mathcal{V}} \approx 4 \pi \frac{\hbar^{2}}{M} a_{s s} \delta(\mathbf{r})+\hat{\mathcal{V}}_{\text {anis }}^{\mathrm{DD}, \mathrm{QQ}}
$$

with

$$
\begin{gathered}
\hat{\mathcal{V}}_{\mathrm{anis}}^{\mathrm{DD}} \Phi(\mathbf{r}) \approx \frac{\hbar^{2}}{M} \frac{a_{s d}^{\mathrm{DD}}}{k^{2}} \sqrt{5} \times \\
\left\{\frac{1}{8} \delta(\mathbf{r})\left[\left(\frac{\partial}{\partial r}\right)^{5} r^{3} \int P_{2}(\cos \theta) \Phi(\mathbf{r}) d \Omega\right]+\right. \\
\left.9 \frac{\delta(r)}{r^{4}} P_{2}(\cos \theta)\left[\frac{\partial}{\partial r}(r \Phi(\mathbf{r}))\right]_{r \rightarrow 0}\right\}
\end{gathered}
$$

for dipolar interactions and

$$
\begin{aligned}
& \hat{\mathcal{V}}_{\mathrm{anis}}^{\mathrm{QQ}} \Phi(\mathbf{r}) \approx \frac{1}{2^{7}} \frac{\hbar^{2}}{M} \frac{a_{s g}^{\mathrm{QQ}}}{k^{2}} \times \\
& \left\{\delta(\mathbf{r})\left[\left(\frac{\partial}{\partial r}\right)^{9} r^{5} \int P_{4}(\cos \theta) \Phi(\mathbf{r}) d \Omega\right]+\right. \\
& \left.5(8 !) \frac{\delta(r)}{r^{6}} P_{4}(\cos \theta)\left[\frac{\partial}{\partial r}(r \Phi(\mathbf{r}))\right]_{r \rightarrow 0}\right\}
\end{aligned}
$$

for quadrupolar interactions. Quantities $\delta(r) / r^{n}$ may be recognized as $n^{\text {th }}$ derivatives of the Dirac delta-function.

The constructed pseudo-potential depends on the relative momentum $k$, i.e. the potential is velocity dependent. In practice, the velocity-dependence is most easily treated in momentum representation and in the next section we evaluate matrix element of the derived pseudopotential in the basis of plane waves.

\section{MATRIX ELEMENT OF ANISOTROPIC PSEUDO-POTENTIAL IN FREE-PARTICLE BASIS}

While considering effects of two-particle interactions on properties of a quantum many-body system, one may require a matrix element of the derived pseudo-potential in free-particle (plane-wave) basis. We define this matrix element as

$$
\begin{aligned}
& \bar{V}\left(\mathbf{k}_{1}, \mathbf{k}_{2}, \mathbf{k}_{1}^{\prime}, \mathbf{k}_{2}^{\prime}\right) \equiv \\
& \frac{1}{(2 \pi)^{6}} \int d \mathbf{r} d \mathbf{r}^{\prime} e^{-i \mathbf{k}_{1} \cdot \mathbf{r}} e^{-i \mathbf{k}_{2} \cdot \mathbf{r}^{\prime}} \hat{\mathcal{V}}\left(\mathbf{r}-\mathbf{r}^{\prime}\right) e^{i \mathbf{k}_{1}^{\prime} \cdot \mathbf{r}} e^{i \mathbf{k}_{2}^{\prime} \cdot \mathbf{r}^{\prime}}
\end{aligned}
$$

The pseudo-potential $\hat{\mathcal{V}}$, Eq. (13), depends on the momentum of relative motion of the interacting pair $\mathbf{k}=$ $\frac{1}{2}\left(\hat{\mathbf{p}}-\hat{\mathbf{p}}^{\prime}\right)$, where $\hat{\mathbf{p}}$ and $\hat{\mathbf{p}}^{\prime}$ are momenta conjugated to $\mathbf{r}$ and $\mathbf{r}^{\prime}$ respectively. To separate center of mass and relative motions, we change the variables to $\mathbf{R}=\left(\mathbf{r}+\mathbf{r}^{\prime}\right) / 2$ and $\mathbf{r}_{12}=\mathbf{r}-\mathbf{r}^{\prime}$. With such a substitution

$$
\begin{gathered}
\bar{V}\left(\mathbf{k}_{1}, \mathbf{k}_{2}, \mathbf{k}_{1}^{\prime}, \mathbf{k}_{2}^{\prime}\right)=\frac{1}{(2 \pi)^{3}} \delta_{\mathbf{k}_{1}^{\prime}+\mathbf{k}_{2}^{\prime}, \mathbf{k}_{1}+\mathbf{k}_{2}}\left(-\frac{\hbar^{2}}{M}\right) \times \\
\sum_{l l^{\prime} m m^{\prime}} \xi_{l m}^{l^{\prime} m^{\prime}}\left(k^{\prime}\right) \int d \mathbf{r}_{12} \exp \left[-i \mathbf{k} \cdot \mathbf{r}_{12}\right] \hat{v}_{l m}^{l^{\prime} m^{\prime}} \exp \left[i \mathbf{k}^{\prime} \cdot \mathbf{r}_{12}\right]
\end{gathered}
$$


Here we introduced two relative momenta

$$
\mathbf{k}=\frac{1}{2}\left(\mathbf{k}_{1}-\mathbf{k}_{2}\right) \text { and } \mathbf{k}^{\prime}=\frac{1}{2}\left(\mathbf{k}_{1}^{\prime}-\mathbf{k}_{2}^{\prime}\right) .
$$

The delta function $\delta_{\mathbf{k}_{1}^{\prime}+\mathbf{k}_{2}^{\prime}}, \mathbf{k}_{1}+\mathbf{k}_{2}$ ensures conservation of the total linear momentum. Further we use partial-wave expansion

$\exp \left[i \mathbf{k}^{\prime} \cdot \mathbf{r}_{12}\right]=4 \pi \sum_{l_{1} m_{1}} i^{l_{1}} j_{l_{1}}\left(k^{\prime} r_{12}\right) Y_{l_{1} m_{1}}^{*}\left(\hat{k}^{\prime}\right) Y_{l_{1} m_{1}}\left(\hat{r}_{12}\right)$

and arrive at

$$
\begin{array}{r}
\int d \mathbf{r}_{12} \exp \left[-i \mathbf{k} \cdot \mathbf{r}_{12}\right] \hat{v}_{l m}^{l^{\prime} m^{\prime}}\left(\mathbf{r}_{12}\right) \exp \left[i \mathbf{k}^{\prime} \cdot \mathbf{r}_{12}\right]= \\
(4 \pi)^{2} i^{l^{\prime}-l} \frac{l+1}{2 l+1}\left(k^{\prime}\right)^{l^{\prime}} k^{l} Y_{l^{\prime} m^{\prime}}^{*}\left(\hat{k}^{\prime}\right) Y_{l m}(\hat{k}) .
\end{array}
$$

Finally, the matrix element of the anisotropic pseudopotential may be expressed in terms of relative momenta as

$$
\begin{aligned}
\bar{V}\left(\mathbf{k}_{1}, \mathbf{k}_{2}, \mathbf{k}_{1}^{\prime}, \mathbf{k}_{2}^{\prime}\right)= & \delta_{\mathbf{k}_{1}^{\prime}+\mathbf{k}_{2}^{\prime}, \mathbf{k}_{1}+\mathbf{k}_{2}} \times \\
& \bar{v}\left(\frac{1}{2}\left(\mathbf{k}_{1}-\mathbf{k}_{2}\right), \frac{1}{2}\left(\mathbf{k}_{1}^{\prime}-\mathbf{k}_{2}^{\prime}\right)\right),
\end{aligned}
$$

with

$$
\begin{aligned}
\bar{v}\left(\mathbf{k}, \mathbf{k}^{\prime}\right)= & -\frac{\hbar^{2}}{M} \frac{1}{\pi} \sum_{l l^{\prime} m m^{\prime}} i^{l^{\prime}-l} \frac{\mathcal{K}_{l m}^{l^{\prime} m^{\prime}}\left(k^{\prime}\right)}{k^{\prime}} \times \\
& \left(\frac{k}{k^{\prime}}\right)^{l} \frac{2 l+2}{2 l+1} Y_{l^{\prime} m^{\prime}}^{*}\left(\hat{k}^{\prime}\right) Y_{l m}(\hat{k}) .
\end{aligned}
$$

Let us once again specialize the discussion to dipolar and quadrupolar interactions. As in Section IV we assume that the dominant anisotropic effect arises due to couplings of $s$ and $d$ partial waves for dipolar interactions and due to mixing of $s$ and $g$ waves for quadrupolar interactions. The corresponding truncated matrix element (30) may be represented as

$$
\bar{v}\left(\mathbf{k}, \mathbf{k}^{\prime}\right) \approx \frac{1}{2 \pi^{2}} \frac{\hbar^{2}}{M}\left\{a_{s s}+\mathcal{F}\left(\mathbf{k}, \mathbf{k}^{\prime}\right)\right\}
$$

with $\mathcal{F}$ replaced by

$\mathcal{F}^{\mathrm{DD}}\left(\mathbf{k}, \mathbf{k}^{\prime}\right)=-a_{s d}^{\mathrm{DD}}\left\{\sqrt{5} P_{2}\left(\cos \theta_{k^{\prime}}\right)+\frac{3}{\sqrt{5}}\left(\frac{k}{k^{\prime}}\right)^{2} P_{2}\left(\cos \theta_{k}\right)^{\text {Mlational Science Foundation. }}\right.$

for dipolar interactions and by

$\mathcal{F}^{\mathrm{QQ}}\left(\mathbf{k}, \mathbf{k}^{\prime}\right)=a_{s g}^{\mathrm{QQ}}\left\{3\left(k^{\prime}\right)^{2} P_{4}\left(\cos \theta_{k^{\prime}}\right)+\frac{10}{3} k^{2}\left(\frac{k}{k^{\prime}}\right)^{2} P_{4}\left(\cos \theta_{k}\right)\right\}$

for quadrupolar interactions. In these expressions $a_{s s}$ is a generalized scattering length (19) and $a_{s d}^{\mathrm{DD}}$ and $a_{s g}^{\mathrm{QQ}}$ are off-diagonal scattering length and volume defined by Eq. (23) and Eq. (24) respectively.
APPENDIX A: OFF-DIAGONAL SCATTERING LENGTH $a_{s d}^{\mathrm{DD}}$ AND SCATTERING VOLUME $a_{s g}^{\mathrm{QQ}}$ IN THE BORN APPROXIMATION

\section{CONCLUSION}

I rigorously derived anisotropic pseudopotential arising in the context of adiabatic collisions of two particles polarized by external field. Such low-energy pseudopotential may be useful in describing collective properties of dilute quantum gases, such as molecules polarized by an external electric field or metastable ${ }^{3} P_{2}$ atoms polarized by magnetic field. The pseudopotential is given by Eq.(13). It is naturally expressed in terms of reactance $(\mathrm{K}-)$ matrix. The potential is non-Hermitian and velocity-dependent. It worth noting that in the derivation I did not require the validity of the Born approximation as in Ref. [5. Rather I followed method of Huang and Yang [- 4 and at large separations demanded the equality of solutions of two-body Schrödinger equation with a full original potential and with a pseudo-potential. Thus, compared to Eq. (5) by Yi and You [5], the derived two-body pseudopotential is expected to be also valid in a vicinity of scattering resonances.

I argued that in most applications the pseudopotential may be represented as a sum of traditional sphericallysymmetric contact term and anisotropic part, Eq. (25). The former contribution may be parameterized by a generalized scattering length (19). We specialized discussion of the anisotropic part of pseudopotential to dipolar and quadrupolar interactions and found that it can be characterized by off-diagonal scattering length $a_{s d}^{\mathrm{DD}}$, Eq. (23), for dipolar interactions and off-diagonal scattering volume $a_{s g}^{\mathrm{QQ}}$, Eq. (24), for quadrupolar interactions. Although in a particular application these parameters should be determined from a solution of multi-channel scattering problem, I have derived $a_{s d}^{\mathrm{DD}}$ and $a_{s g}^{\mathrm{QQ}}$ in the Born approximations. Keeping in mind many-body applications, I have also derived two-body matrix-element in the plane-wave basis, Eq. (30). Thus in this work I have rigorously derived anisotropic pseudo-potential for polarized dilute quantum gases and investigated its properties.

\section{Acknowledgments}

I would like to thank Eite Tiesinga for stimulating discussions and $\mathrm{Li}$ You and $\mathrm{Su} \mathrm{Yi}$ for comments on manuscript. This work was supported in part by the ,

Here we obtain expressions for reactance $\left(\mathrm{K}^{-}\right)$matrix in the Born approximation. Using the derived K-matrix we estimate off-diagonal scattering length $a_{s d}^{\mathrm{DD}}$ and scattering volume $a_{s g}^{\mathrm{QQ}}$ for dipolar (DD) and quadrupolar (QQ) interactions. 
The full solution of the Schrödinger equation (6) may be represented as

$$
\Phi(\mathbf{r})=\sum_{l m} Y_{l m}(\theta, \phi) \frac{u_{l m}(r)}{k r} .
$$

It can be shown that the radial functions $u_{l m}(r)$ satisfy the following system of coupled differential equations

$$
\begin{gathered}
\left\{\frac{d^{2}}{d r^{2}}-\frac{l(l+1)}{r^{2}}+k^{2}\right\} u_{l m}(r)= \\
2 \mu \sum_{l^{\prime} m^{\prime}}\left\langle l m|V| l^{\prime} m^{\prime}\right\rangle u_{l^{\prime} m^{\prime}}(r),
\end{gathered}
$$

with

$$
\left\langle l m|V| l^{\prime} m^{\prime}\right\rangle(r)=\int d \Omega Y_{l m}^{*}(\Omega) V(\mathbf{r}) Y_{l^{\prime} m^{\prime}}(\Omega) .
$$

It is convenient to introduce regular and irregular solutions of homogeneous radial equations

$$
\begin{aligned}
F_{l}(k r) & =k r j_{l}(k r), \\
G_{l}(k r) & =-k r n_{l}(k r)
\end{aligned}
$$

and corresponding standing-wave Green's function

$$
g_{l}\left(r, r^{\prime}\right)=-\frac{1}{k}\left\{\begin{array}{l}
G_{l}\left(k r^{\prime}\right) F_{l}(k r), r<r^{\prime} \\
G_{l}(k r) F_{l}\left(k r^{\prime}\right), r>r^{\prime}
\end{array} .\right.
$$

Using these definitions, solutions to the system of inhomogenous equations (A2) regular at $r=0$ may be represented as
By comparing with Eq. (7) and Eq. (9), we arrive at expression for elements of K-matrix in the Born approximation

$$
\mathcal{K}_{l m}^{l^{\prime} m^{\prime}} \approx-\frac{2 \mu k}{\hbar^{2}} \int_{0}^{\infty} r^{2} j_{l}(k r) j_{l^{\prime}}(k r)\left\langle l m|V| l^{\prime} m^{\prime}\right\rangle d r .
$$

The Born approximation generally does not hold for low-energy atomic collisions, since realistic interactions are singular at small $r$. However, for dipolar interactions You and co-workers 22, 23] found numerically that away from resonances Born approximation works well for offdiagonal matrix elements. Keeping this observation in mind, below we derive off-diagonal scattering length and volume introduced in the main body of the paper. These parameters were defined as low-energy limits

$$
\begin{aligned}
& a_{s d}^{\mathrm{DD}}=-\lim _{k \rightarrow 0} \frac{\left[\mathcal{K}^{\mathrm{DD}}\right]_{00}^{20}(k)}{k}, \\
& a_{s g}^{\mathrm{QQ}}=-\lim _{k \rightarrow 0} \frac{\left[\mathcal{K}^{\mathrm{QQ}}\right]_{00}^{40}(k)}{k^{3}}
\end{aligned}
$$

for dipolar (DD) and quadrupolar (QQ) interactions. In Section 1 the DD and QQ interactions were parameterized as

$$
\begin{aligned}
V_{\mathrm{DD}} & =\frac{C_{3}}{r^{3}} P_{2}(\cos \theta), \\
V_{\mathrm{QQ}} & =\frac{C_{5}}{r^{5}} P_{4}(\cos \theta) .
\end{aligned}
$$

$u_{l m}(r)=\alpha_{l m} F_{l}(k r)+\int_{0}^{\infty} d r^{\prime} g_{l}\left(r, r^{\prime}\right)\left[2 \mu \sum_{l^{\prime} m^{\prime}}\left\langle l m|V| l^{\prime} m^{\prime}\right\rangle u_{l^{\prime} m^{\prime}}\right.$ sing $\left.\left.^{\prime}\right)\right]$ these definitions and Eq. (A5), we arrive at

where constants $\alpha_{l m}$ are chosen to satisfy some boundary conditions. In the spirit of Born approximation we may find solution of integral equations (A4) iteratively starting from

$$
u_{l m}(r) \approx \alpha_{l m} F_{l}(k r) .
$$

In the lowest order in $V$ at large $r$ one obtains

$u_{l m}(r) \rightarrow \alpha_{l m} F_{l}(k r)+G_{l}(k r) \alpha_{l m} \sum_{l^{\prime} m^{\prime}}\left[-\frac{2 \mu}{k} \int_{0}^{\infty} d r^{\prime} F_{l}\left(k r^{\prime}\right)\right.$

It is worth emphasizing that these results were derived in the Born approximation. In general case, to obtain parameters entering anisotropic pseudo-potential (13) one

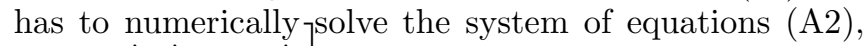

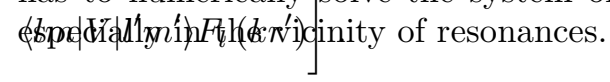

[1] Y. N. Demkov and V. N. Ostrovsky, Zero-range Potentials Method in Atomic Physics (Plenum, New York, 1988).

[2] F. Dalfovo, S. Giorgini, L. P. Pitaevskii, and S. Stringari, Rev. Mod. Phys. 71, 463 (1999).

[3] A. J. Leggett, Rev. Mod. Phys. 73, 307 (2001).

[4] K. Huang and C. N. Yang, Phys. Rev. A 105, 767 (1957).

[5] S. Yi and L. You, Phys. Rev. A 63, 053607/1 (2000).
[6] S. Yi and L. You, Phys. Rev. A 66, 013607/1 (2002).

[7] S. Giovanazzi, A. Görlitz, and T. Pfau, Phys. Rev. Lett. 89, 130401 (2002).

[8] L. Santos, G. V. Shlyapnikov, P. Zoller, and M. Lewenstein, Phys. Rev. Lett. 85, 1791 (2000).

[9] K. Góral, K. Rzążewski, and T. Pfau, Phys. Rev. A 61, 051601/1 (2000).

[10] K. Góral and L. Santos, Phys. Rev. A 66, 023613 (2002). 
[11] K. Góral, L. Santos, and M. Lewenstein, Phys. Rev. Lett. 88, 170406 (2002).

[12] S. Giovanazzi, D. O'Dell, and G. Kurizki, Phys. Rev. Lett. 88, 130402 (2002).

[13] K. Góral, B.-G. Englert, and K. Rzążewski, Phys. Rev. A 63, 033606 (2001).

[14] M. A. Baranov, M. S. Mar'enko, V. S. Rychkov, and G. V. Shlyapnikov, Phys. Rev. A 66, 013606 (2002).

[15] J. Kim, B. Friedrich, D. P. Katz, D. Patterson, J. D. Weinstein, R. DeCarvalho, and J. M. Doyle, Phys. Rev. Lett. 78, 3665 (1997).

[16] J. D. Weinstein, R. deCarvalho, J. Kim, D. Patterson, B. Friedrich, and J. M. Doyle, Phys. Rev. A 57, R3173 (1998).

[17] A. S. Bell, J. Stuhler, S. Locher, S. Hensler, J. Mlynek, and T. Pfau, Europhys. Lett. 45, 156 (1999).

[18] A. Derevianko, S. G. Porsev, S. Kotochigova, E. Tiesinga, and P. S. Julienne, e-print:physics/0210076.

[19] A. L. Fetter and J. D. Walecka, Quantum Theory of Many-particle Systems (McGraw-Hill, 1971).

[20] N. F. Mott and H. S. W. Massey, The theory of atomic collisions (Oxford University Press, London, 1965), 3rd ed.

[21] S. Geltman, Topics in atomic collision theory (Krieger Pub. Co., Florida, 1997).

[22] M. Marinescu and L. You, Phys. Rev. Lett. 81, 4596 (1998).

[23] B. Deb and L. You, Phys. Rev. A 64, 022717/1 (2001). 